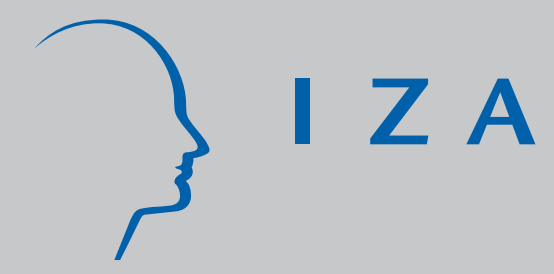

IZA DP No. 2598

A New Continuous Distribution and Two New Families of Distributions Based on the Exponential

Guillermina J asso

Samuel Kotz

February 2007 


\title{
A New Continuous Distribution and Two New Families of Distributions Based on the Exponential
}

\author{
Guillermina Jasso \\ New York University and IZA \\ Samuel Kotz \\ George Washington University
}

Discussion Paper No. 2598

February 2007

\author{
IZA \\ P.O. Box 7240 \\ 53072 Bonn \\ Germany \\ Phone: +49-228-3894-0 \\ Fax: +49-228-3894-180 \\ E-mail: iza@iza.org
}

\begin{abstract}
Any opinions expressed here are those of the author(s) and not those of the institute. Research disseminated by IZA may include views on policy, but the institute itself takes no institutional policy positions.

The Institute for the Study of Labor (IZA) in Bonn is a local and virtual international research center and a place of communication between science, politics and business. IZA is an independent nonprofit company supported by Deutsche Post World Net. The center is associated with the University of Bonn and offers a stimulating research environment through its research networks, research support, and visitors and doctoral programs. IZA engages in (i) original and internationally competitive research in all fields of labor economics, (ii) development of policy concepts, and (iii) dissemination of research results and concepts to the interested public.
\end{abstract}

IZA Discussion Papers often represent preliminary work and are circulated to encourage discussion. Citation of such a paper should account for its provisional character. A revised version may be available directly from the author. 


\section{ABSTRACT}

\section{A New Continuous Distribution and Two New Families of Distributions Based on the Exponential}

Recent work on social status led to derivation of a new continuous distribution based on the exponential. The new variate, termed the ring(2)-exponential, in turn leads to derivation of two closely-related new families of continuous distributions, which we call the mirrorexponential and the ring-exponential. Both the standard exponential and the ring(2)exponential are special cases of both the new families. In this paper, we first focus on the ring(2)-exponential, describing its derivation and examining its properties, and next introduce the two new families, describing their derivation and initiating exploration of their properties. The mirror-exponential arises naturally in the study of status; the ring-exponential arises from the mathematical structure of the ring(2)-exponential. Both have potential for broad application in diverse contexts across science and engineering, including the physical and social sciences as well as finance, information processing, and communication. Within sociobehavioral contexts, the new mirror-exponential may have application to the problem of approximating the form and inequality of the wage distribution.

JEL Classification: C02, C16, D31, D6, I3

Keywords: continuous univariate distributions, Erlang distribution, general Erlang distribution, gamma distribution, general gamma distribution, folded distributions, Gini coefficient, social status, social inequality, wage function, wage distribution, wage inequality

Corresponding author:

Guillermina Jasso

Department of Sociology

New York University

295 Lafayette Street, 4th Floor

New York, NY 10012-9605

USA

E-mail: gj1@nyu.edu

\footnotetext{
* We thank participants at the annual Winter Conference of the Methodology Section of the American Sociological Association and at the annual Group Processes Conference, especially Joseph Berger, David Wagner, and Murray Webster, for their valuable comments; and we thank the editor and the anonymous referee for their close reading and valuable comments. We also gratefully acknowledge New York University and George Washington University for their intellectual and financial support. This paper is forthcoming in Statistica Neerlandica.
} 


\section{INTRODUCTION}

This paper examines a recently derived continuous distribution based on the exponential, termed the ring(2)-exponential, and introduces two closely-related new families of continuous distributions to which it leads, termed the mirror-exponential and the ring-exponential. The ring(2)-exponential and the mirror-exponential arise naturally in the study of social status; the ring-exponential is a natural generalization of the $\operatorname{ring}(2)$-exponential based on its mathematical structure.

As will be seen below, the mathematical structure of the new variates' associated functions are evocative of tree rings, in one case, and mirror images, in the other, thus suggesting the names we chose for them.

Both new variate families have potential for application in a wide range of contexts across science and engineering.

\section{GENESIS OF THE RING(2)-EXPONENTIAL}

Consider the process by which persons accord to each other, and expect from each other, a given measure of status - or, as it is variously called, prestige, respect, deference, esteem, honor (Zelditch 1968; Sennett 2003). Status is represented by positive numbers (the higher the number, the greater the status), and is thought to arise from individuals' personal quantitative characteristics, such characteristics as wealth, beauty, intelligence, or any of a multitude of skills (musical, mathematical, athletic, public-speaking, military, and so on), where the individual's standing on these quantitative characteristics is represented by his or her relative rank within a group or population. ${ }^{1}$

The mathematical foundation for studying status was laid by Berger, Cohen, and Zelditch

1 Personal quantitative characteristics are those characteristics of which there can be more or less, or on which an individual may rank higher or lower. The quantitative characteristics include both cardinal characteristics (such as wealth, land, and livestock) and ordinal characteristics (such as beauty and intelligence). 
(1966), Berger, Fisek, Norman, and Zelditch (1977), Goode (1978), and Sørensen (1979), as discussed below.

Goode (1978) observed that status increases at an increasing rate, and Sørensen (1979) proposed a specific mathematical function for studying the status of occupations. Noting that the function proposed by Sørensen (1979) satisfies Goode’s (1978) convexity condition, Jasso (2001) applied it to the status of individuals:

$$
S=\ln \left(\frac{1}{1-r}\right), 0<r<1,
$$

where $S$ denotes status and $r$ denotes the individual's relative rank on a quantitative characteristic. $^{2}$

Status processes are pervasive in the social life, and understanding their operation is a central task for social science. Status affects the happiness of individuals and the harmony of societies, touching virtually every area of the human experience. Across millenia and across cultures and civilizations, from religious writings to the Greek epics to the novels of Jane Austen and Nikolai Gogol, the long reach of status is at work. Abraham, desperate to buy a plot of land to bury Sarah in the Promised Land, bows in deference to the Hittites (Genesis 23); the artistic treasures left at Persepolis document status, the satraps of Persia paying respect to the King, a senior official saluting Xerxes, and so on (Curtis and Tallis 2005). Surrounded by status hierarchies, the young Jesus imagines a world where "the last will be first, and the first last" (Matthew 19:30) - an idea he probably learned from his mother, who before his birth proclaimed, “depósuit poténtes de sede et exaltávit humiles” (Magnificat, Luke 1:52) -- and the older Don Quixote comforts Sancho, "When we sleep, we are all equal" (Book II, Ch 43) - a thought Cervantes probably learned from Vives (Introduction to Wisdom, item \#59).

Scientific understanding of status has been hampered due to several longstanding

${ }^{2}$ Note the use of the logarithmic function, which evokes information-theoretic concepts (Shannon 1948). 
unresolved issues, including: (i) the emergence of status; (ii) how to distinguish between, and measure, the status of persons and the status of characteristics; (iii) how to measure overall status inequality; and (iv) how to measure the status gap between subgroups (e.g., between women and men, or between ethnic groups).

Building on the status function in (1), Jasso (2001) proposed a framework which integrates the insights of several literatures and a theory which provides direction for solving the longstanding unresolved issues and which yields a rich variety of testable implications, including: (i) in a two-subgroup society, the least advantaged from both subgroups gain status from discrimination, and the most advantaged from both subgroups lose status from discrimination; (ii) the status gap between two subgroups increases with the relative size of the disadvantaged subgroup; (iii) in subgroup conflict, it is more difficult to prevent defections from the bottom subgroup than from the top subgroup; and (iv) opponents of discrimination are outnumbered by proponents of discrimination.

Many of the deduced implications depend on properties of the distribution of status. Thus, analytic focus quickly expands from the individual to encompass variation in status across a collection of individuals.

As Sørensen (1979) had pointed out, the status function in (1) is in fact the quantile function of the standard exponential variate, and hence the status distribution in this first case in which status is generated by a single characteristic is already known, as are all its properties, including status inequality. For example, in a large population tending to infinity, the mean and variance of the status distribution are both equal to 1 , and the median is $\ln (2)=.6931$. . The status distribution has a Gini's mean difference of 1 and a Gini coefficient of .5 - so that status inequality is greater than the inequality of many of the quantitative characteristics which generate 
it (the Gini coefficient in U.S. personal income, for example, does not reach .5). ${ }^{3,4}$

But the simple one-characteristic case represented in the status function in (1) is only the first step in representing the operation of status. Consistent with the large classical literature, status may be generated by several quantitative characteristics (or goods) simultaneously, for example, not only by wealth but also by beauty, or not only by military daring but also by horsemanship (as in the case of Julius Caesar). ${ }^{5}$ Jasso (2001:101-102) theorized that when status is generated by two or more characteristics, the multiple-good status function is a weighted average of the single-good status functions, where the weights represent the importance of each good. For example, an individual or society may accord status based on wealth, beauty, and musical skill, with the three goods weighted $.7, .2$, and .1, respectively, while another individual or society may accord status based on the same three goods but weight them $.1, .45$, and .45 , respectively, and so on.

Earlier, Berger, Cohen, and Zelditch (1966:44), had noted that negatively associated characteristics would reduce status inequality (a relation subsequently established for small groups by Humphreys and Berger (1981)), and Berger, Fisek, Norman, and Zelditch (1977:126127) had proposed the principle of organized subsets (applied below) for dealing with multiple negatively associated characteristics. In the multiple-good case, however, the goods need not

3 For definitions and formulas of the Gini measures, see Gini (1913-1914) or any of numerous expositions, such as Kleiber and Kotz (2003).

4 A new and potentially important sociobehavioral question arises immediately: Under what conditions does the status distribution have lower inequality than the underlying quantitative characteristics? The terrain for further analysis is wide open. For example, consider the question that the Greeks might have asked: If status and the underlying quantitative characteristics are in competition for the individual's attention, which dominates in a population marked by anhedonia? And which in a population marked by analgēsia? And how effective are changes in wealth inequality at producing changes in status inequality? These questions are especially timely, given the current increases in income inequality and wealth inequality in the United States, some Western countries, and, recently, in Russia and China.

${ }^{5}$ Quantitative characteristics of which more is preferred to less are called goods; quantitative characteristics of which less is preferred to more are called bads. 
only be positively or negatively associated; they may also be independent. ${ }^{6}$

Accordingly, Jasso (2001) derived the status distribution for three further special cases: (i) multiple goods, all perfectly positively associated; (ii) multiple goods, equally weighted, independent; and (iii) two goods, equally weighted, perfectly negatively associated. ${ }^{7}$ The status distribution remains a standard exponential in the first case, becomes an Erlang in the second case (a gamma with positive integer shape parameter), and assumes a new form in the third case, a form which Jasso (2001:122) termed "Unnamed" but which we here term the ring(2)exponential because, as will be seen, the mathematical form of its quantile function is evocative of tree rings. Some basic formulas and graphs were presented for the two-goods cases (Jasso 2001:109, 122). As will be seen below, the ring(2)-exponential turns out to be a particular case of two new variate families, and so does the standard exponential. ${ }^{8}$

Immediately there were some interesting results, especially concerning inequality in the status distribution. Status inequality is lower in the two-goods-independent case than in the single-good case and lower still in the two-goods-negative case, thus extending to large populations modeled by probability distributions the link between negatively-associated goods and inequality reduction first discussed by Berger, Cohen, and Zelditch (1966:44). Indeed, the

${ }^{6}$ A parallel line of development can be discerned in the study of justice - like status one of the fundamental sociobehavioral forces (Jasso in press). Jasso (1980:6, 10-11, 14, 30) proposed multi-good justice evaluations and reported multi-good justice evaluation distributions for the case of independent goods, and Jasso (1983:264) added the case of negatively associated goods.

${ }^{7}$ Perfect positive association denotes the case in which each individual has the same relative rank on all the goods generating status. Perfect negative association of two goods denotes the case in which the rank ordering in one good is exactly the reverse of the rank ordering in the other good; thus one ranking is the conjugate ranking of the other (Kotz, Johnson, and Read 1982:145).

${ }^{8}$ It may happen that a quantitative characteristic possesses two or more aspects or "faces", as suggested by the anonymous referee. For example, beauty may generate two manifestations, beauty as a signal of genetic endowment and beauty as visual aesthetic. In such case, each aspect is treated as a distinct good. The distinct goods may then, like all goods, be independent or positively associated or negatively associated. 
lower extreme value, which is zero in the single-good case, the multiple-goods-positive case, and the two-goods-independent cases, approaches in the two-goods-negative case $\ln (2)$, which is the median in the first two cases! $!^{9}$

Moreover, the Gini coefficient in the multiple-goods-equally-weighted-independent case diminishes quickly, from .5 in the single-good case to .375 in the two-good case, .3125 in the three-good case, and so on. The formula for the Gini coefficient in the case of the Erlang distribution is well known (see, for example, McDonald and Jensen (1979:856) and Kleiber and Kotz (2003:164)):

$$
G=\frac{\Gamma(g+1 / 2)}{\Gamma(g+1) \sqrt{\pi}},
$$

where $g$ denotes the number of goods. Thus, the Gini coefficient approaches zero as $g \rightarrow \infty$.

The ring(2)-exponential variate promises even faster strides toward equality, leading to a new research question: For given $g$ number of goods, what is the inequality differential between the $g$-goods-independent case and the g-goods-negative case? Before this question can be answered it is necessary to give meaning to the g-goods-negative case and to obtain the first of the two new variate families, tasks to which we turn in section 4 after completing our examination of the ring(2)-exponential in the next section. Further, we note that it is straightforward to show that the status distribution in the case of multiple independent goods unequally weighted is a general Erlang distribution, also known as a general gamma distribution (McGill and Gibbon (1965); Johnson, Kotz, and Balakrishnan (1994:552)).

9 Note that the case of two negatively associated goods in the study of justice and status has the same mathematical structure as the case of negative assortative mating, which has been thought at least since Plato ( $\underline{\text { Laws }}$, Book VI) to reduce inequality. 


\section{THE RING(2)-EXPONENTIAL: THE STATUS DISTRIBUTION WHEN TWO GOODS ARE PERFECTLY NEGATIVELY ASSOCIATED AND EQUALLY WEIGHTED}

Consider the status arising from relative rank $r$ on the first good (given in expression (1)). Introduce a second good, perfectly negatively associated with the first, where perfect negative association is defined by the conjugate ranking (Kotz, Johnson, and Read 1982:145). Then the relative rank on the second good is given by $(1-r)$. It follows that each person's new status score $S^{*}$ in the case where the two negatively associated goods are equally weighted is given by:

$$
\begin{aligned}
S^{*} & =\frac{1}{2}\left[\ln \left(\frac{1}{1-r}\right)+\ln \left(\frac{1}{r}\right)\right] \\
& =\ln \left(\frac{1}{\sqrt{r(1-r)}}\right)=\ln \left(\frac{1}{\sqrt{r-r^{2}}}\right),
\end{aligned}
$$

where $r$ is the relative rank on the first good.

By inspection, it is obvious that, unlike the single-good formula in (1), the formula in (3) for status in the two-goods-equally-weighted-negative case is not a quantile function; indeed, $S^{*}$ is not even an increasing function of $r$. Accordingly, the next step is to obtain the quantile function of the new two-goods-negative status distribution.

Further inspection and analysis of the personal status formula in (3) show (as do graphs) that $S^{*}$ is U-shaped and symmetric about .5. Thus, making use of procedures from the study of folded distributions (Read 1983), it follows that $\alpha$ the argument of the quantile function for the new variate is given by:

$$
\alpha=2(r-1 / 2)=2 r-1,
$$

so that

$$
r=\frac{1+\alpha}{2},
$$

which is then substituted into the expression for personal status in (3), yielding the quantile function for the ring(2)-exponential variate representing status in the two-goods-equally- 
weighted-negative case, as reported by Jasso (2001:122):

$$
Q(\alpha)=\ln \left(\frac{2}{\sqrt{1-\alpha^{2}}}\right) .
$$

Note that the quantile function of the ring(2)-exponential variate, given in (6), represents in fact the "new" status function, where $\alpha$ is the new $r$ (the individual's relative rank) in the new composite distribution arising from the two negatively-associated goods.

The density and distribution function for the ring(2)-exponential, as reported by Jasso (2001:122), are given by:

$$
f(x)=\frac{4 e^{-x}}{\sqrt{e^{2 x}-4}}
$$

and

$$
F(x)=\sqrt{1-\frac{4}{e^{2 x}}} .
$$

Table 1 provides the principal properties of the ring(2)-exponential and, for comparison, of the standard exponential and the Erlang with parameter 2, which characterize status in the onegood case and the two-goods-independent-equally-weighted cases, respectively. Figures 1-3 depict the $\mathrm{CDF}$, PDF, and quantile function of the ring(2)-exponential and, for contrast, the standard exponential and the Erlang.

- Table 1 about here -

- Figures 1-3 about here -

Note that the Erlang is not truly an exponential, given that it contains an extra factor, a polynomial or monomial in $x$. The graphs show vividly the family resemblance between the standard exponential and the ring(2)-exponential, a resemblance not shared by the Erlang.

As shown in Table 1 and in the Figures and as already noted, the ring(2)-exponential has its lower extreme value at $\ln (2)$. Of course, the mean is the same as in all the status distributions: 1. Its Gini coefficient is $\ln (2)-1 / 2$, or approximately .193, almost half that for the two-goodsindependent case, .375 . Thus, in the study of status, the possibility that people may value 
negatively associated goods may serve as a key to reducing status inequality.

Recall that the quantile function of the standard exponential at value $\alpha$ is:

$$
Q(\alpha)=\ln \left(\frac{1}{1-\alpha}\right)
$$

corresponding to the density function,

$$
f(x)=e^{-x},
$$

and the cumulative distribution function,

$$
F(x)=1-e^{-x}
$$

Comparing (6), (7), and (8) with (9), (10), and (11), one observes that (6), (7), and (8) represent a one-step generalization of the standard exponential described in (9), (10), and (11). Indeed, the quantile function presents three instances of this one-step generalization; the exponential's quantile function in (9), relative to the ring(2)-exponential's quantile function in (6), involves (i) the "first power" instead of the "second power", (ii) the "first root" instead of the "second root", and (iii) the coefficient 1 instead of 2.

The shift from a trio of $1 \mathrm{~s}$ in the standard exponential to a trio of $2 \mathrm{~s}$ in the new variate is evocative of a tree adding rings, and for that reason we call the new variate the ring(2)exponential.

\section{GENERALIZING THE RING(2)-EXPONENTIAL TO THE NEW MIRROR-EXPONENTIAL FAMILY: MULTIPLE GOODS NEGATIVELY ASSOCIATED}

To define the case of $g$ goods negatively associated, we use the principle of organized subsets proposed by Berger, Fisek, Norman, and Zelditch (1977:126-127), applying it as follows: (i) there are $g$ goods, arranged in two subsets; (ii) within each subset, all the goods are perfectly positively associated; and (iii) the two subsets are perfectly negatively associated with each other. For example, suppose that a society values three goods, beauty, wealth, and musical skill; beauty and wealth are perfectly positively associated, and the set of beauty and wealth is perfectly 
negatively associated with musical skill.

It is obvious that if $g$ is even and the two subsets each contain $g / 2$ goods, the situation is equivalent to the case of two goods equally weighted, as above; i.e., the variate is the ring(2)exponential. In this section we establish that all other cases of multiple goods negatively associated (as just defined) are equivalent to the case of two goods unequally weighted and we derive and describe the new family of distributions, of which the ring(2)-exponential is a special case.

\subsection{Equivalence of Two-Goods-Unequally-Weighted and Multiple-Goods-Negatively-Associated}

\section{Excluding the Case Where $g$ is Even and Equally Divided between the Two Subsets}

Let each of the two subsets of goods have $P$ and $Q$ goods, respectively, so that $P+Q=g$. Then, as with the two-good case, using the conjugate ranking leads to the new formula for status:

$$
S^{*}=\ln \left(\frac{1}{\sqrt[g]{r^{P}(1-r)^{Q}}}\right),
$$

where $r$ is the individual's relative rank on the first subset of goods.

Now define $p=P / g$ and $q=Q / g$, so that $p$ and $q$ are weights and sum to one. (Of course, $p$ and $q$ are continuous and not necessarily confined only to rational numbers.) Thus, it can be seen that the case of $g$ goods is equivalent to a special case of the general case of two goods unequally weighted. The formula for $S^{*}$ for the weighted case is given by:

$$
S^{*}=\ln \left(\frac{1}{r^{p}(1-r)^{q}}\right)=\ln \left(\frac{1}{r^{p}(1-r)^{1-p}}\right)=-p \ln (r)-(1-p) \ln (1-r) .
$$

In the expressions shown in (13), $p$ and $(1-p)$ are reflections of each other about .5 , as are $r$ and $(1-r)$. These are but the first instances of reflections that recur in the associated functions and formulas of the new variate family, evoking a mirror, and thus we name this family the mirror-exponential.

\subsection{Immediate Properties of the New Mirror-Exponential}

Some important properties of the new mirror-exponential follow directly from its 
description.

1. Mean of the new mirror-exponential. The mean of the new variate is 1 . This is evident from theorem on the mean of a linear combination of variables.

2. Lower extreme value of the new mirror-exponential.

2.1. Formula for the lower extreme value. By analyzing (13), we obtain the lower extreme value of the new variate. Differentiating (13) with respect to $r$, we set the derivative to zero to find the critical point $r_{0}$, then solve for $S^{*}$ at $r_{0}$. This procedure yields the result that the critical point occurs at $r$ equals $p$ and yields the lower extreme value (LEV):

$$
\mathrm{LEV}=\ln \left(\frac{1}{p^{p}(1-p)^{1-p}}\right)=-p \ln (p)-(1-p) \ln (1-p)
$$

Observe again how the factors in the denominator of the first formula (and the terms in the second formula) evoke a mirror.

2.2. Relation between LEV and the weight parameter. Differentiating (14) with respect to $p$, we find that the LEV has an inverted-U shape and is symmetric about .5. Thus, the LEV reaches its zenith when the weight $p$ is exactly one-half. The new family of distributions has a nonzero minimum which varies nonmonotonically with $p$, reaching its peak when $p$ equals onehalf. In the status application, minimum status is at its highest when the valued goods are either (i) two goods equally weighted and negatively associated (the case highlighted in Jasso 2001, which we are calling the ring(2)-exponential), or (ii) $g$ goods, such that $g$ is even and divided into two equal subsets of goods, each subset containing perfectly positively associated goods and the two subsets perfectly negatively associated with each other.

2.3. Peak LEV. The peak LEV, occurring when the weight $p$ is one-half, is $\ln (2)$, or approximately .693. As already noted, given that $\ln (2)$ is the median of the exponential (which characterizes the status distribution in the one-good and multiple-goods-positively-associated cases), this particular member of the new mirror-exponential family $(p=1 / 2)$ is remarkable and of great interest to students of status inequality. 


\subsection{Derivation of the New Mirror-Exponential}

Using procedures from the study of folded distributions, we obtain the quantile function of the new mirror-exponential. Briefly, generalizing the procedure described in section 3 , we define two new distributions, one each for the regions to the left and to the right of the rank $r$ at the lowest point of $S^{*}$, that is, when $r$ equals $p$, and then combine them. $S^{*}$ is U-shaped in $r$; when $p$ is one-half, $S^{*}$ is symmetric about $r$; otherwise, it is asymmetric. Using procedures for asymmetrically folded distributions, we write expressions for $\alpha$ the argument of the quantile function and then obtain the corresponding expressions for $r$.

For the distribution to the right of $r, \alpha$ the argument of the quantile function is equal to the ratio of $(r-p)$ to $(1-p)$, that is, to the fraction whose numerator is $(r-p)-$ the distance between the relative rank $r$ in the right region in (13) and the folding point $p$ - and whose denominator is $(1-p)$ - the proportion of the distribution in the region to the right of the folding point:

$$
\alpha=\frac{r-p}{1-p}
$$

so that

$$
r=\alpha+p(1-\alpha),
$$

which is then substituted into the expression for personal status in (13), yielding the quantile function for the distribution to the right of $r$.

For the distribution to the left of $r, \alpha$ is equal to the ratio of $(p-r)$ to $p$, that is, to the fraction whose numerator is $(p-r)$ - the distance between the relative rank $r$ in the left region in (13) and the folding point $p$ - and whose denominator is $p$ - the proportion of the distribution in the region to the left of the folding point:

$$
\alpha=\frac{p-r}{p},
$$

so that

$$
r=p(1-\alpha),
$$

which, again, is substituted into the expression for personal status in (13), yielding the quantile 
function for the distribution to the left of $r$.

The two distributions are then combined, weighted by $p$ and $(1-p)$, respectively. This procedure yields the quantile function for the new mirror-exponential:

$$
\begin{aligned}
& (1-p) \ln \left(\frac{1}{[\alpha+p(1-\alpha)]^{p}[1-\alpha-p(1-\alpha)]^{1-p}}\right)+p \ln \left(\frac{1}{[p(1-\alpha)]^{p}[1-p(1-\alpha)]^{1-p}}\right) \\
= & (1-p) \ln \left(\frac{1}{[\alpha+p(1-\alpha)]^{p}[(1-p)(1-\alpha)]^{1-p}}\right)+p \ln \left(\frac{1}{[p(1-\alpha)]^{p}[1-p(1-\alpha)]^{1-p}}\right) .
\end{aligned}
$$

Again, note how the components of the quantile function in (19) are almost mirror images of each other.

The expressions above simplify to the following expression for the quantile function of the new mirror-exponential:

$$
-\left(p-p^{2}\right) \ln \left\{\alpha+\left[\left(p-p^{2}\right)(1-\alpha)^{2}\right]\right\}-(1-p)^{2} \ln [(1-p)(1-\alpha)]-p^{2} \ln [p(1-\alpha)] .
$$

Comparing the expression in (20) for the $\mathrm{QF}$ of the new mirror-exponential to the $\mathrm{QF}$ for Tukey's lambda distribution,

$$
\frac{\alpha^{\lambda}-(1-\alpha)^{\lambda}}{\lambda}
$$

it may be plausible to conjecture that closed-form expressions for the distribution function and the density function of the mirror-exponential do not exist. If this turns out to be the case, then the new mirror-exponential joins Tukey's lambda distribution in this somewhat exclusive club. It is more common for the QF to defy analytic expression than for the CDF, and the Erlang is an example. Of course, knowledge of any one of the three basic associated functions (PDF, CDF, QF) is sufficient to represent the distribution and obtain its main parameters (albeit numerically in some cases).

\subsection{Gini Coefficient in the New Mirror-Exponential}

The quantile function in (20) enables derivation of the formula for the Gini coefficient:

$$
G=\frac{-p^{3} \ln (p)-(1-p)^{3} \ln (1-p)}{p(1-p)}-\frac{1}{2}
$$


As shown, and as expected from the QF, the Gini coefficient is a function solely of the weight parameter $p$. Differentiating (21) with respect to $p$ reveals that the Gini coefficient is U-shaped with respect to $p$, being symmetric about .5 , its lowest point. The Gini's magnitude at its lowest point is, as already known from the ring(2)-exponential, $\ln (2)-1 / 2$, or approximately .193. The Gini's highest magnitude is .5, occurring when $p$ equals zero or one. Figure 4 depicts the Gini coefficient in the new mirror-exponential.

- Figure 4 about here -

Thus, in the new variate the minimum value ranges from zero to $\ln (2)$, and the Gini coefficient ranges from .193 to .5. The highest minimum and the lowest inequality occur when $p$ equals $1 / 2$. In the status application, minimum status is highest and status inequality is lowest when the valued goods are (i) two equally-weighted negatively-associated goods or (ii) several goods, arranged in two subsets such that within subset the goods are perfectly positively associated and such that the two subsets are equally weighted and perfectly negatively associated.

Earlier we posed the question whether inequality is higher or lower in the independent or negatively-associated case. That question can now be answered. Because the Gini diminishes to zero in the Erlang but only to .193 in the new variate, inequality can be lower in the goodsindependent case than in the goods-negative case. Whether it is or not depends on particular cases.

Consider the case of equally-weighted goods. In the independent case, Gini declines monotonically, from $1 / 2$ (for one good) to zero, as the number of goods increases. In the negative case, Gini oscillates, starting at $1 / 2$ (again for one good), dropping to its minimum of .193 at two goods, rising to .224 at 3 goods (in subsets of 1 and 2), and thereafter displaying a within- $g$ pattern such that it declines as the number of goods becomes more evenly divided between the two subsets, reaching its minimum of .193 when the number of goods is even and the two subsets each contain half the goods. Table 2 displays the values of the Gini coefficient for this case of equally-weighted goods.

- Table 2 about here - 
Several rather surprising results are immediate:

1. When the number of goods is less than or equal to 4 , the new mirror-exponential's Gini coefficient is always smaller than the Erlang's Gini coefficient.

2. When the number of goods is between 5 and 8 , inclusive, one or the other variate can have the smaller Gini; however, when the number of goods is 6 or 8 , and each subset contains half the goods ( 3 and 4 , respectively), the new mirror-exponential always has the smaller Gini.

3. When the number of goods is 9 or greater, the Erlang variate always has the smaller Gini coefficient.

Thus, in the status application, status inequality is not necessarily lower when the goods are negatively-associated than when the goods are independent. Indeed, the number of goods and the subset configuration play important parts. Moreover, in complex societies in which people value many goods - beauty, wealth, athletic skill, military skill, musical skill, card-playing skill, etc. - independence of the goods reduces status inequality more effectively than negativelyassociated sets of (positively-associated) goods. These results contribute to a sharper understanding of the exact way in which the association between goods affects status inequality, a question that has challenged students of status since the original conjecture by Berger, Cohen, and Zelditch (1966:44). New research questions arise immediately concerning the natural association or independence among goods, a topic of burgeoning interest in evolutionary biology. ${ }^{10}$

At this juncture, we begin collecting features and properties of the new mirrorexponential family, reporting them in Table 3.

- Table 3 about here -

\subsection{Median in the New Mirror-Exponential}

The quantile function also enables derivation of the formula for the median:

${ }^{10}$ These results are for equally-weighted goods. Future work will contrast the independent and negatively-associated cases for unequally-weighted goods. 


$$
-p(1-p) \ln \left(-p^{2}+p+2\right)-(1-p)^{2} \ln (1-p)-p^{2} \ln (p)+\ln (2) .
$$

Again, the median is solely a function of the weight parameter $p$. Differentiating (23) with respect to $p$ reveals that the median has an inverted-U shape, being symmetric about .5, its highest point. The smallest value of the median, which occurs at the zero and one extremes of $p$, is $\ln (2)$. The highest value of the median, already known from the ring(2)-exponential, is $\ln (4 / \sqrt{3})$, or approximately .837 .

Thus, adding to the results above, in the new mirror-exponential, the highest median, highest minimum, and lowest inequality occur when $p$ equals $1 / 2$, as in the ring(2)-exponential.

\subsection{The Standard Exponential and the Ring(2)-Exponential As Bookends of the New Mirror-Exponential}

As discussed in the preceding sections and reported in Tables 1 and 3, the standard exponential and the ring(2)-exponential are not only particular cases of the new mirrorexponential family. They are special particular cases, marking the boundaries of variation and operating as bookends to the entire collection of all possible members of the mirror-exponential family. The standard exponential represents the high-inequality pole - minimum of zero, median of $\ln (2)$, Gini coefficient of .5 - and the ring(2)-exponential represents the low-inequality pole minimum of $\ln (2)$, median of .837, Gini coefficient of .193.

To illustrate, we show in Figure 5 the graphs of the quantile function for members of the mirror-exponential family with parameter $p$ equal to $0, .1, .2, .3, .4$, and .5 . By symmetry, family members with parameter $p$ are identical to members with parameter $(1-p)$, so that members with $p$ equal to $.6, .7, .8, .9$, and 1 are identical to members represented in the figure.

- Figure 5 about here -

Thus, the new mirror-exponential family and the Erlang family (including the general Erlang) are jointly sufficient for representing all possible distributions of status, whether based on one good or multiple goods and, if multiple goods, whether equally or unequally weighted and whether the goods are independent or perfectly positively associated or perfectly negatively associated. Future work might further explore the cases of imperfect positive and negative 
association.

\subsection{A Possible New Application of the New Mirror-Exponential: Wage Function, Wage Distribution, and Wage Inequality}

There is much interest in modeling income distributions (Kleiber and Kotz 2003). We note that the new mirror-exponential family of distributions may provide an appropriate model. Consider the problem of wage determination. Most discussions approach it as a problem of establishing a wage function, where the inputs are quantitative variables such as schooling and effort. But economic theory also provides for the possibility of a different approach, namely, an approach based on supply and demand. If attributes are more desirable the more scarce they are, then perhaps the more appropriate wage function would have as inputs relative ranks rather than amounts.

Accordingly, the exponential would provide the basic wage function, now with a parameter for the salary budget, here represented by the mean $\mu$ :

$$
W=\mu \ln \left(\frac{1}{1-r}\right),
$$

where $W$ denotes the wage and $r$ is the individual's relative rank. As with status, it is straightforward to include any number of characteristics and to specify their weights and relations to each other.

In this approach, the wage function in (24) applies within a wage-setting context - firm, industry-occupation sector, government service, military, etc. Thus, within wage-setting context the wage distribution would be either exponential, Erlang, general Erlang, or mirror-exponential, depending on the number of wage-relevant characteristics and their weights and associations. One payoff would be an orderly and systematic account of the etiology and magnitude of wage inequality.

Of course, it would not be trivial if the same account also yields the form of the wage distribution for the more routine entities in which wage functions and wage distributions are usually estimated, such as cities, states, and countries. For such entities, the wage distribution 
would be a mixture composed of the distributions or subdistributions describing the various complete or partial wage-setting contexts spanned by the entity. It is not difficult to imagine that the mixtures produce a dazzling diversity of shapes -- and thus the seeming defiance of realworld income distributions to straightforward characterization.

In this radically different approach, the theoretical and empirical tasks would shift to prediction and assessment, respectively, of the relevant wage-setting contexts, the number and identity of wage-relevant characteristics in each type of wage-setting context and their weights and associations. And the statistical tasks would shift to description of the theoretically possible mixtures.

\section{GENERALIZING THE RING(2)-EXPONENTIAL TO THE NEW RING-EXPONENTIAL FAMILY \\ 5.1. Derivation of the New Ring-Exponential}

Look again at the quantile function for the ring(2)-exponential, given in expression (6). The number 2 appears three times, in the numerator of the fraction, in the exponent of $\alpha$, and in the exponent of the denominator of the fraction (embedded in the square root). Above we observed that the ring(2)-exponential is thus a one-step generalization of the standard exponential, in which the number 1 appears three times instead of the number 2 . It is evident that the ring(2)-exponential can immediately be generalized to a larger family, which we may call the ring-exponential, in which the number 2 is generalized to the parameter $k$. The generalization can be conceptualized in (at least) two ways. First, replace the standard exponential's parameter " 1 " by $k$ an integer. Second, multiply the standard exponential's parameter " 1 " by the sequence of integers $k$.

The quantile function of this new variate is thus given by:

$$
Q(\alpha)=\ln \left(\frac{k}{\sqrt[k]{1-\alpha^{k}}}\right)=\ln \left[k\left(1-\alpha^{k}\right)^{-1 / k}\right]
$$


Here we are treating the parameter $k$ as an integer but it may be further generalized in the future. The distribution function is then given by:

$$
F(x)=\sqrt[k]{1-\frac{k^{k}}{e^{k x}}}=\left[1-k^{k} e^{-k x}\right]^{1 / k}
$$

and the density function by:

$$
f(x)=\frac{k^{k} e^{-x}}{\sqrt[k]{e^{k x}-k^{k}}}=k^{k} e^{-x}\left(e^{k x}-k^{k}\right)^{\frac{1-k}{k}} .
$$

Figures 6,7 , and 8 depict the distribution function, density function, and quantile function, respectively, for ten members of the ring-exponential family, with values of $k$ from 1 to 10.

- Figures 6, 7, and 8 about here -

As can readily be seen in the formulas and graphs, not only is the ring(2)-exponential a particular case of the ring-exponential (with $k=2$ ) - contrast the ring(2)-exponential's quantile function in (6) with expression (25) -- but so also is the standard exponential (with $k=1$ ) - look again at the standard exponential's quantile function in (9).

Note that the generalization of the standard exponential to the ring(2)-exponential and finally to the ring-exponential is visible in all its associated functions, not only in the quantile function, as highlighted above. Consider the density function. The ring-exponential stems from the standard exponential via generalizing the kernel function $e^{-x}$ by introducing a multiplier involving the $k$ th root of a function of $e^{k x}$.

Note also that, like the mirror-exponential, the ring-exponential draws its basic form from the exponential - its density has a single mode at the lower extreme value, and there are no points of inflection in either the density or the quantile function (or, equivalently, the CDF).

Finally, note that the ring-exponential is not the only possible generalization of the ring(2)-exponential based on its mathematical structure. The anonymous referee kindly proposed an alternate generalization, a generalization leading to a new family which, like the ringexponential, has the standard exponential and the ring(2)-exponential as special cases but whose 
other members differ from those in the ring-exponential. The QFs for the two variates differ in that the term $k$ in the numerator of the fraction in the ring-exponential's QF (expression (25)) is replaced by the term $\left[\left(2^{\wedge}(k-1)\right]\right.$. The curves for the two QFs are similar in shape but differ by a multiplicative constant, with this constant increasing as $k$ increases. A formula with a power of $k$ in the numerator is indeed appealing. Moreover, the existence of this alternate generalization suggests the possibility of a still larger family of which both this alternate generalization and the ring-exponential introduced here are special cases. This new possibility merits sustained inquiry, a task we leave to future research.

The new ring-exponential joins the mirror-exponential in Table 3, and we collect its basic functions and other properties.

\subsection{Properties of the New Ring-Exponential}

Lower extreme value of the ring-exponential. By inspection of the quantile function, it is immediately evident that the lower extreme value of the new ring-exponential is $\ln (k)$. The larger

the parameter $k$, the higher the minimum of the variate. Table 4 presents values of the LEV for $k$ equal 1 to 10 , in steps of 1 , and 10 to 60 , in steps of 5 .

- Table 4 about here -

Median of the ring-exponential. Using the quantile function, we obtain the median:

$$
\ln \left[2 k\left(2^{k}-1\right)^{-1 / k}\right] \text {. }
$$

In the special case of $k$ an integer, the median increases with the parameter $k$. Values of the median are also reported in Table 4.

Mean of the ring-exponential. A closed-form expression for the mean (and other moments) so far eludes us. Using numerical methods, we obtained the sequence of means for $k$ equal 1 to 10, in steps of 1, and 10 to 60, in steps of 5; these are reported in Table 4.

\subsection{Gini Coefficient in the New Ring-Exponential}

As with the mean, we used numerical methods to obtain values of the Gini coefficient for the full set of values of the parameter $k$ in Table 4. The Gini coefficient declines quickly, from .5 when $k$ equals one, to .193 when $k$ equals two - as already known from our work with the 
standard exponential and the ring(2)-exponential - to remarkably low values: between .01 and .1 for $k$ between 3 and 7; between .001 and .01 for $k$ between 8 and 20; between .0001 and .001 for $k$ between 25 and 60. This drastic reduction in inequality as $k$ increases suggests that the ring-exponential becomes degenerate.

\subsection{Degeneracy in the New Ring-Exponential}

The figures in Table 4 jointly indicate that the ring-exponential approaches degeneracy as $k$ increases. Because closed-form expressions exist for the minimum and the median and because one of the components of the formula for the median has a well-known limit:

$$
\lim _{k \rightarrow \infty}\left(2^{k}-1\right)^{1 / k}=2
$$

it is not difficult to prove that, as $k$ increases, the limit of the difference between the minimum and the median is zero:

$$
\lim _{k \rightarrow \infty} \ln (k)-\ln \left[2 k\left(2^{k}-1\right)^{-1 / k}\right]=0
$$

The quantile function provides another way to assess and visualize the approach to degeneracy. It is straightforward to show that the limit of the difference between the minimum and the $\mathrm{QF}$, for all values of $\alpha$, is zero:

$$
\lim _{k \rightarrow \infty} \ln (k)-\ln \left[k\left(1-\alpha^{k}\right)^{-1 / k}\right]=0 .
$$

As shown in Figure 8, as the parameter $k$ increases from 1 to 10, the graphs become increasingly flatter. In fact, as Table 4 and the limits in (30) and (31) show, as $k$ increases beyond 10, the flatness becomes more pronounced.

\section{CONCLUDING NOTE}

The exponential distribution is a towering distribution. Like the normal, it gives form and voice to an astonishing range of phenomena, and effortlessly multiplies its kin - the double exponential, the doubly exponential, the hyperexponential, the hypoexponential, the sliding 
exponential, the inverse exponential, and so on. In this paper, we examined one new variate based on the standard exponential, the ring(2)-exponential which arises in the study of social status, and introduced two new variate families it inspires - the ring-exponential which generalizes it and the mirror-exponential which arises naturally in deeper analysis of status.

The two new variate families arise from the exponential not by adding parameters but in rather special and distinctive ways. The mirror-exponential arises via modeling a substantive phenomenon - status - which in its simplest form is represented by the standard exponential and in its more elaborate forms leads to the Erlang, general Erlang, and mirror-exponential families. The ring-exponential arises via generalizing the associated mathematical functions; this generalization is visible in many functions, including, as we have seen, the quantile function and the density function, where the ring-exponential generalizes the kernel function $e^{-x}$, introducing a multiplier involving the $k$ th root of a function of $e^{k x}$.

By way of summary, we depict in Figure 9 the quantile functions for four members of each of the two new distributional families, including the two common to both families - the standard exponential and the ring(2)-exponential. The graphs in Figure 9 show clearly that the two families have a common origin - displaying the form of the exponential and sharing the standard exponential as a common foundation -- while at the same time showing that the two families are distinct. Because both families begin with the standard exponential, but the mirrorexponential ends in the infancy, as it were, of the ring-exponential, there is a sort of "morphing," such that the ring(2)-exponential stands at the end of one era and the start of another. And because the Gini coefficient, which declines within the mirror-exponential family from .5 to .193, starts a precipitous decline as the ring-exponential leaves behind the mirror-exponential, the two families may serve to model, and to understand, a wide range of processes which show the tendency to become degenerate. In the social life, the march to equality may be a case in point.

$$
\text { - Figure } 9 \text { about here - }
$$

Much could be learned in future both by further analysis of these variates and by application across a range of contexts in science and engineering. 


\section{REFERENCES}

Berger, Joseph, Bernard P. Cohen, and Morris Zelditch, Jr. 1966. "Status Characteristics and Expectation States.” Pp. 29-46 in Joseph Berger, Morris Zelditch, Jr., and Bo Anderson (eds.), Sociological Theories in Progress, Volume 1. Boston: Houghton Mifflin.

, M. Hamit Fisek, Robert Z. Norman, and Morris Zelditch, Jr. 1977. Status

Characteristics and Social Interaction. New York: Elsevier.

Curtis, John, and Nigel Tallis (eds.). 2005. Forgotten Empire: The World of Ancient Persia. Catalogue of exhibit at the British Museum. London, UK: British Museum Press.

Gini, Corrado. 1913-1914. "Sulla Missura della Concentrazione e della Variabilità dei Caratteri." Atti del Reale Istituto Veneto di Scienze, Lettere ed Arti 53 (Part 2):12031248.

Goode, William J. 1978. The Celebration of Heroes: Prestige as a Control System. Berkeley, CA: University of California Press.

Humphreys, Paul, and Joseph Berger. 1981. "Theoretical Consequences of the Status Characteristics Formulation.” American Journal of Sociology 86:953-983.

Jasso, Guillermina. 1980. "A New Theory of Distributive Justice." American Sociological Review 45:3-32. . 1983. "Social Consequences of the Sense of Distributive Justice: Small-Group Applications." Pp. 243-294 in David M. Messick and Karen S. Cook (eds.), Theories of Equity: Psychological and Sociological Perspectives. New York: Praeger. . 2001. "Studying Status: An Integrated Framework." American Sociological Review 66:96-124. . In press. "A New Unified Theory of Sociobehavioral Forces." European Sociological Review.

Johnson, Norman L., Samuel Kotz, and N. Balakrishnan. 1994. Continuous Univariate Distributions, Volume 1. Second Edition. New York, NY: Wiley.

Kleiber, Christian, and Samuel Kotz. 2003. Statistical Size Distributions in Economics and 
Actuarial Sciences. Hoboken, NJ: Wiley.

Kotz, Samuel, Norman L. Johnson, and Campbell B. Read. 1982. "Conjugate Ranking." P. 145

in Samuel Kotz, Norman L. Johnson, and Campbell B. Read (eds.). Encyclopedia of Statistical Sciences, Volume 2. New York: Wiley.

McDonald, James B., and Bartell C. Jensen. 1979. “An Analysis of Some Properties of Alternative Measures of Income Inequality Based on the Gamma Distribution Function.” Journal of the American Statistical Association 74:856-860.

McGill, William J., and John Gibbon. 1965. "The General-Gamma Distribution and Reaction Times." Journal of Mathematical Psychology 2:1-18

Plato. 1952. The Dialogues of Plato. Translated by Benjamin Jowett. Chicago: Britannica.

Read, Campbell B. 1983. "Folded Distributions." P. 160-161 in Samuel Kotz, Norman L. Johnson, and Campbell B. Read (eds.). Encyclopedia of Statistical Sciences, Volume 3. New York: Wiley.

Sennett, Richard. 2003. Respect in a World of Inequality. New York: Norton.

Shannon, Claude E. 1948. "A Mathematical Theory of Communication.” Bell System Technical Journal 27:379-423,623-656.

Sørensen, Aage B. 1979. "A Model and a Metric for the Analysis of the Intragenerational Status Attainment Process." American Journal of Sociology 85:361-384.

Zelditch, Morris, Jr. 1968. "Status, Social." Pp. 250-257 in David L. Sills (ed.), International Encyclopedia of the Social Sciences, Volume 15. New York: Macmillan. 
Table 1. Associated Functions, Moments, and Other Properties of the Ring(2)-Exponential Distribution and the Exponential and Erlang Distributions Which Arise in the Study of Social Status

\begin{tabular}{|c|c|c|c|c|}
\hline \multirow[b]{2}{*}{ Feature/Property } & \multirow[t]{2}{*}{ One Good } & \multicolumn{3}{|c|}{ Two Goods, Equally Weighted } \\
\hline & & $\begin{array}{c}\text { Positively } \\
\text { Associated }\end{array}$ & Independent & $\begin{array}{l}\text { Negatively } \\
\text { Associated }\end{array}$ \\
\hline Variate & $\begin{array}{c}\text { Standard } \\
\text { Exponential }\end{array}$ & $\begin{array}{c}\text { Standard } \\
\text { Exponential }\end{array}$ & Erlang & $\begin{array}{c}\text { Ring }(2)- \\
\text { Exponential }\end{array}$ \\
\hline Support & $\mathrm{x}>0$ & $\mathrm{x}>0$ & $x>0$ & $x>\ln (2)$ \\
\hline PDF & $e^{-x}$ & $e^{-x}$ & $4 x e^{-2 x}$ & $\frac{4 e^{-x}}{\sqrt{e^{2 x}-4}}$ \\
\hline $\mathrm{CDF}$ & $1-e^{-x}$ & $1-e^{-x}$ & $1-\left[e^{-2 x}(2 x+1)\right]$ & $\sqrt{1-\frac{4}{e^{2 x}}}$ \\
\hline QF & $\ln \left(\frac{1}{1-\alpha}\right)$ & $\ln \left(\frac{1}{1-\alpha}\right)$ & --- & $\ln \left(\frac{2}{\sqrt{1-\alpha^{2}}}\right)$ \\
\hline Raw Moments & $\mu_{n}^{*}=n !$ & $\mu_{n}^{*}=n !$ & $\left(\frac{1}{2}\right)^{n}(1+n) !$ & --- \\
\hline $1 \mathrm{st}$ & 1 & 1 & 1 & 1 \\
\hline 2nd & 2 & 2 & 1.5 & 1.18 \\
\hline $3 \mathrm{rd}$ & 6 & 6 & 3 & 1.73 \\
\hline 4 th & 24 & 24 & 7.5 & 3.27 \\
\hline 5 th & 120 & 120 & 22.5 & 7.82 \\
\hline 6th & 720 & 720 & 78.75 & 22.95 \\
\hline Mean & 1 & 1 & 1 & 1 \\
\hline Variance & 1 & 1 & .5 & .178 \\
\hline Median & $\ln (2)$ & $\ln (2)$ & .839 & $\ln (4 / \sqrt{3}) \approx .837$ \\
\hline Mode & 0 & 0 & .5 & $\ln (2)$ \\
\hline Skewness & 2 & 2 & $\sqrt{2} \approx 1.414$ & 2.63 \\
\hline Kurtosis & 9 & 9 & 6 & 13.1 \\
\hline Gini coefficient & .5 & .5 & .375 & $\ln (2)-1 / 2 \approx .193$ \\
\hline
\end{tabular}


Table 2. Gini Coefficient, Equally Weighted Goods

\begin{tabular}{|c|c|c|c|}
\hline \multicolumn{2}{|c|}{ Goods } & \multicolumn{2}{|c|}{ Gini Coefficient } \\
\hline Number & $\begin{array}{c}\text { Subset } \\
\text { Configuration }\end{array}$ & $\begin{array}{c}\text { Erlang } \\
\text { (Goods } \\
\text { Independent) } \\
\end{array}$ & $\begin{array}{c}\text { Mirror-Exponential } \\
\text { (Goods Negatively } \\
\text { Associated) } \\
\end{array}$ \\
\hline 2 & 1 and 1 & .375 & .193 \\
\hline 3 & 1 and 2 & .3125 & .224 \\
\hline 4 & 1 and 3 & .273 & .263 \\
\hline 4 & 2 and 2 & .273 & .193 \\
\hline 5 & 1 and 4 & .246 & .295 \\
\hline 5 & 2 and 3 & .246 & .204 \\
\hline 6 & 1 and 5 & .226 & .319 \\
\hline 6 & 2 and 4 & .226 & .224 \\
\hline 6 & 3 and 3 & .226 & .193 \\
\hline 7 & 1 and 6 & .209 & .339 \\
\hline 7 & 2 and 5 & .209 & .244 \\
\hline 7 & 3 and 4 & .209 & .199 \\
\hline 8 & 1 and 7 & .196 & .355 \\
\hline 8 & 2 and 6 & .196 & .263 \\
\hline 8 & 3 and 5 & .196 & .210 \\
\hline 8 & 4 and 4 & .196 & .193 \\
\hline 9 & 1 and 8 & .185 & .368 \\
\hline 9 & 2 and 7 & .185 & .280 \\
\hline 9 & 3 and 6 & .185 & .224 \\
\hline 9 & 4 and 5 & .185 & .197 \\
\hline 10 & 1 and 9 & .176 & .379 \\
\hline 10 & 2 and 8 & .176 & .295 \\
\hline 10 & 3 and 7 & .176 & .237 \\
\hline 10 & 4 and 6 & .176 & .204 \\
\hline 10 & 5 and 5 & .176 & .193 \\
\hline
\end{tabular}

Notes: The goods-negatively-associated case is specified as follows: The goods are arranged in two subsets. Within each subset, the goods are perfectly positively associated. The two subsets are perfectly negatively associated. 
Table 3. Associated Functions, Moments, and Other Properties of Two New Families of Continuous Distributions, the Mirror-Exponential and the Ring-Exponential

\begin{tabular}{|c|c|c|}
\hline Feature/Property & Mirror-Exponential & Ring-Exponential \\
\hline Genesis & modeling status & $\begin{array}{l}\text { mathematical generalization of the } \\
\text { ring(2)-exponential variate }\end{array}$ \\
\hline Parameter & $p: \quad 0 \leq p \leq 1$ & $k$ an integer \\
\hline Support & $x \geq \ln \left(\frac{1}{p^{p}(1-p)^{1-p}}\right)$ & $x \geq \ln (k)$ \\
\hline PDF & --- & $k^{k} e^{-x}\left(e^{k x}-k^{k}\right)^{\frac{1-k}{k}}$ \\
\hline $\mathrm{CDF}$ & --- & {$\left[1-k^{k} e^{-k x}\right]^{1 / k}$} \\
\hline QF & $\begin{array}{c}-\left(p-p^{2}\right) \ln \left\{\alpha+\left[\left(p-p^{2}\right)(1-\alpha)^{2}\right]\right\} \\
-(1-p)^{2} \ln [(1-p)(1-\alpha)] \\
-p^{2} \ln [p(1-\alpha)]\end{array}$ & $\ln \left[k\left(1-\alpha^{k}\right)^{-1 / k}\right]$ \\
\hline Mean & 1 & --- \\
\hline Median & $\begin{array}{c}-p(1-p) \ln \left(-p^{2}+p+2\right) \\
-(1-p)^{2} \ln (1-p) \\
-p^{2} \ln (p)+\ln (2)\end{array}$ & $\ln \left[2 k\left(2^{k}-1\right)^{-1 / k}\right]$ \\
\hline Mode & $\mathrm{LEV}: \ln \left(\frac{1}{p^{p}(1-p)^{1-p}}\right)$ & LEV: $\ln (k)$ \\
\hline Gini coefficient & $\frac{-p^{3} \ln (p)-(1-p)^{3} \ln (1-p)}{p(1-p)}-\frac{1}{2}$ & --- \\
\hline Special cases & $\begin{array}{c}\text { at } p=0 \text { or } 1: \text { Exponential } \\
\text { at } p=.5 \text { : } \operatorname{Ring}(2) \text {-Exponential }\end{array}$ & $\begin{array}{c}\text { at } k=1: \text { Exponential } \\
\text { at } k=2: \operatorname{Ring}(2) \text {-Exponential }\end{array}$ \\
\hline
\end{tabular}


Table 4. Minimum, Median, Mean, and Gini Coefficient of the Ring-Exponential Distribution, as Parameter $k$ Increases

\begin{tabular}{|c|c|c|c|c|}
\hline $\boldsymbol{k}$ & $\begin{array}{c}\text { Minimum } \\
\ln (k)\end{array}$ & $\begin{array}{c}\text { Median } \\
\ln \left[2 k\left(2^{k}-1\right)^{-1 / k}\right]\end{array}$ & Mean & Gini Coefficient \\
\hline 1 & 0 & .693 & 1 & .5 \\
\hline 2 & .693 & .837 & 1 & .193 \\
\hline 3 & 1.099 & 1.143 & 1.247 & .0839 \\
\hline 4 & 1.386 & 1.402 & 1.474 & .0448 \\
\hline 5 & 1.609 & 1.616 & 1.667 & .0273 \\
\hline 6 & 1.792 & 1.794 & 1.833 & .0182 \\
\hline 7 & 1.946 & 1.947 & 1.976 & .0129 \\
\hline 8 & 2.079 & 2.080 & 2.103 & .00957 \\
\hline 9 & 2.197 & 2.197 & 2.216 & .00736 \\
\hline 10 & 2.303 & 2.303 & 2.318 & .00581 \\
\hline 15 & 2.708 & 2.708 & 2.715 & .00235 \\
\hline 20 & 2.996 & 2.996 & 3.000 & .00123 \\
\hline 25 & 3.219 & 3.219 & 3.221 & .000751 \\
\hline 30 & 3.401 & 3.401 & 3.403 & .000500 \\
\hline 35 & 3.555 & 3.555 & 3.557 & .000355 \\
\hline 40 & 3.689 & 3.689 & 3.690 & .000264 \\
\hline 45 & 3.807 & 3.807 & 3.807 & .000203 \\
\hline 50 & 3.912 & 3.912 & 3.913 & .000161 \\
\hline 55 & 4.007 & 4.007 & 4.008 & .000130 \\
\hline 60 & 4.094 & 4.094 & 4.095 & .000108 \\
\hline
\end{tabular}




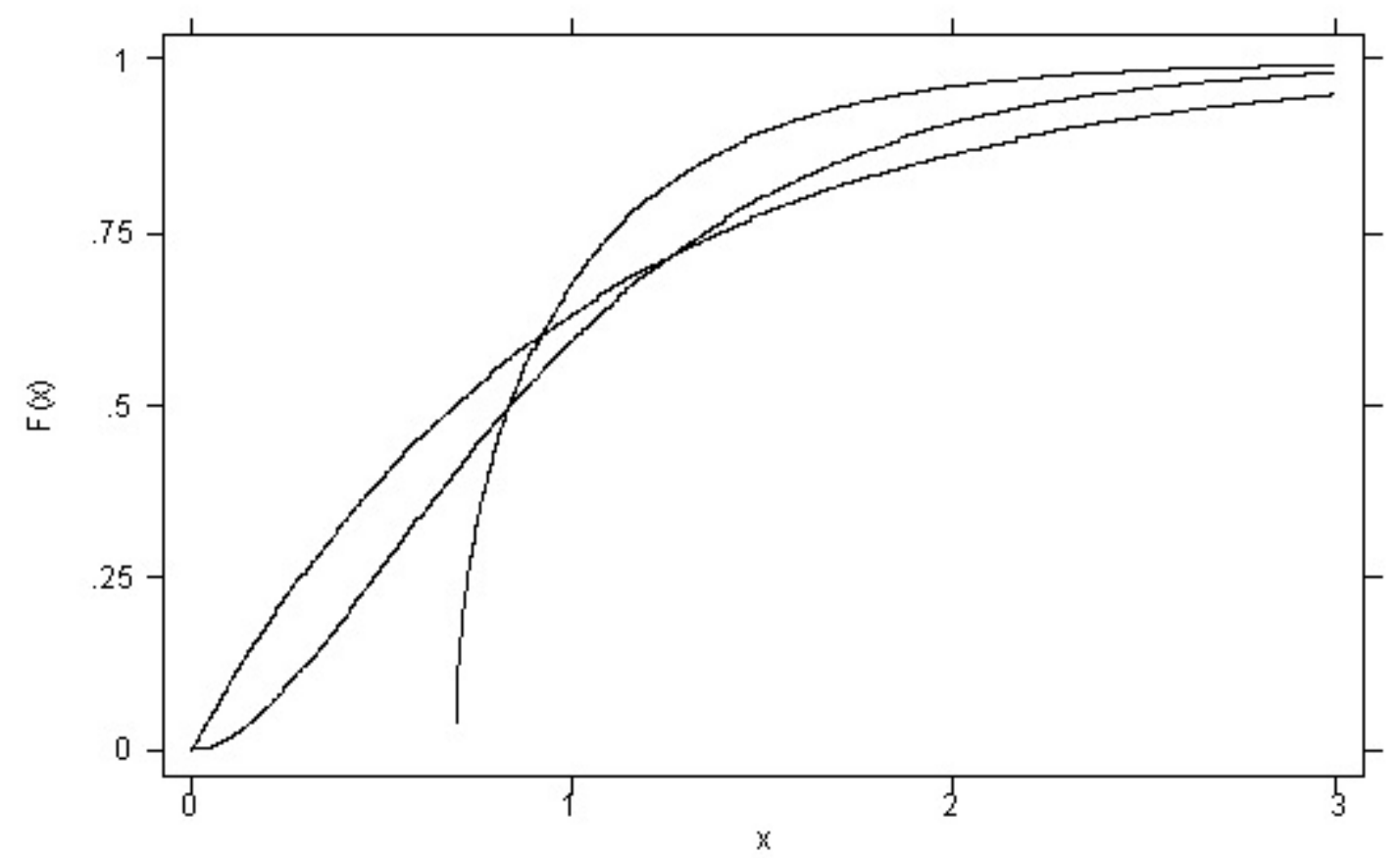

Figure 1. CDF of the Standard Exponential, Erlang, and Ring(2)Exponential Variates (from left to right in the leftmost area of grid) 


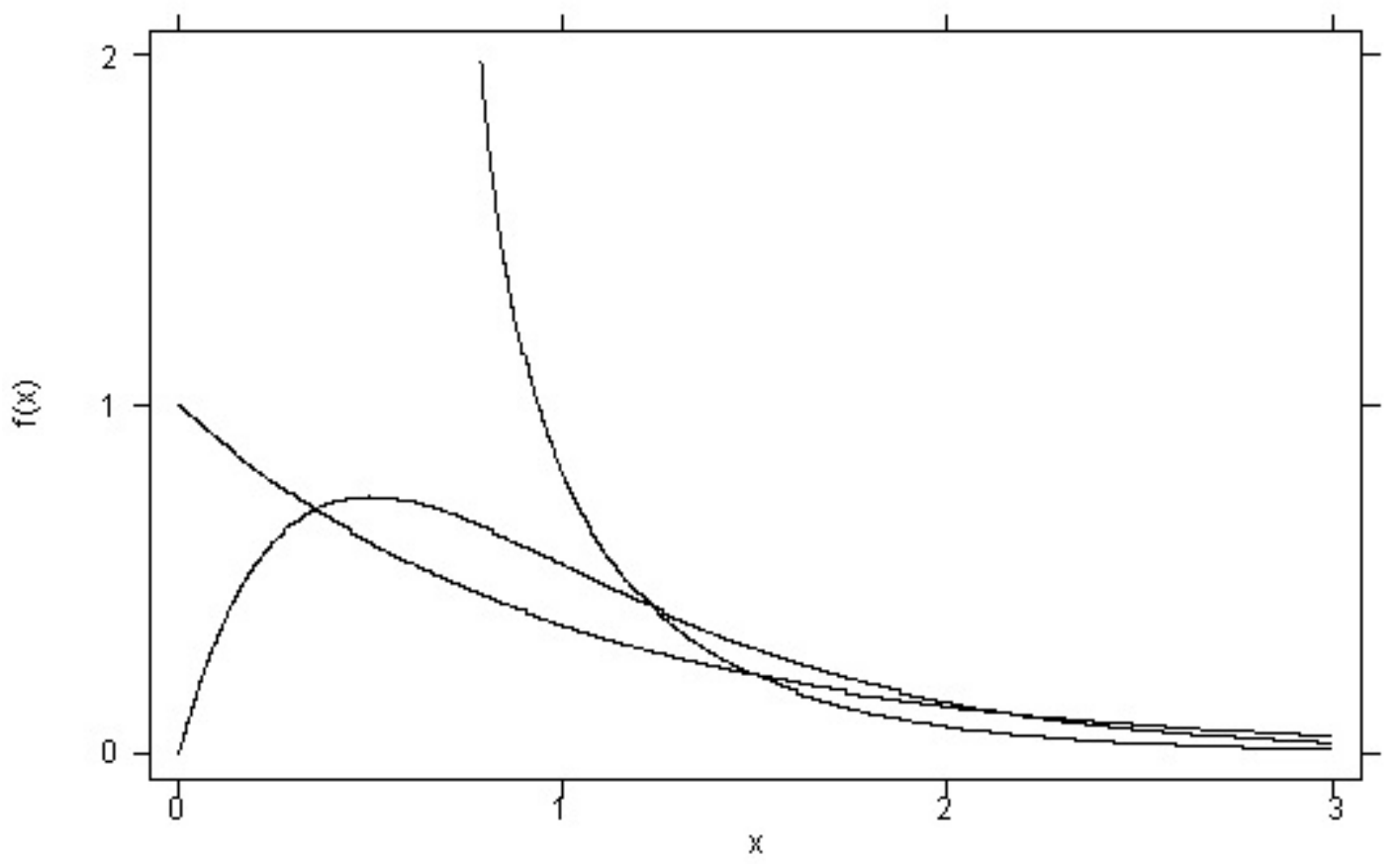

Figure 2. PDF of the Standard Exponential, Erlang, and Ring(2)Exponential Variates. The standard exponential has its mode at zero, and the ring(2)-exponential at $\ln (2)$. 


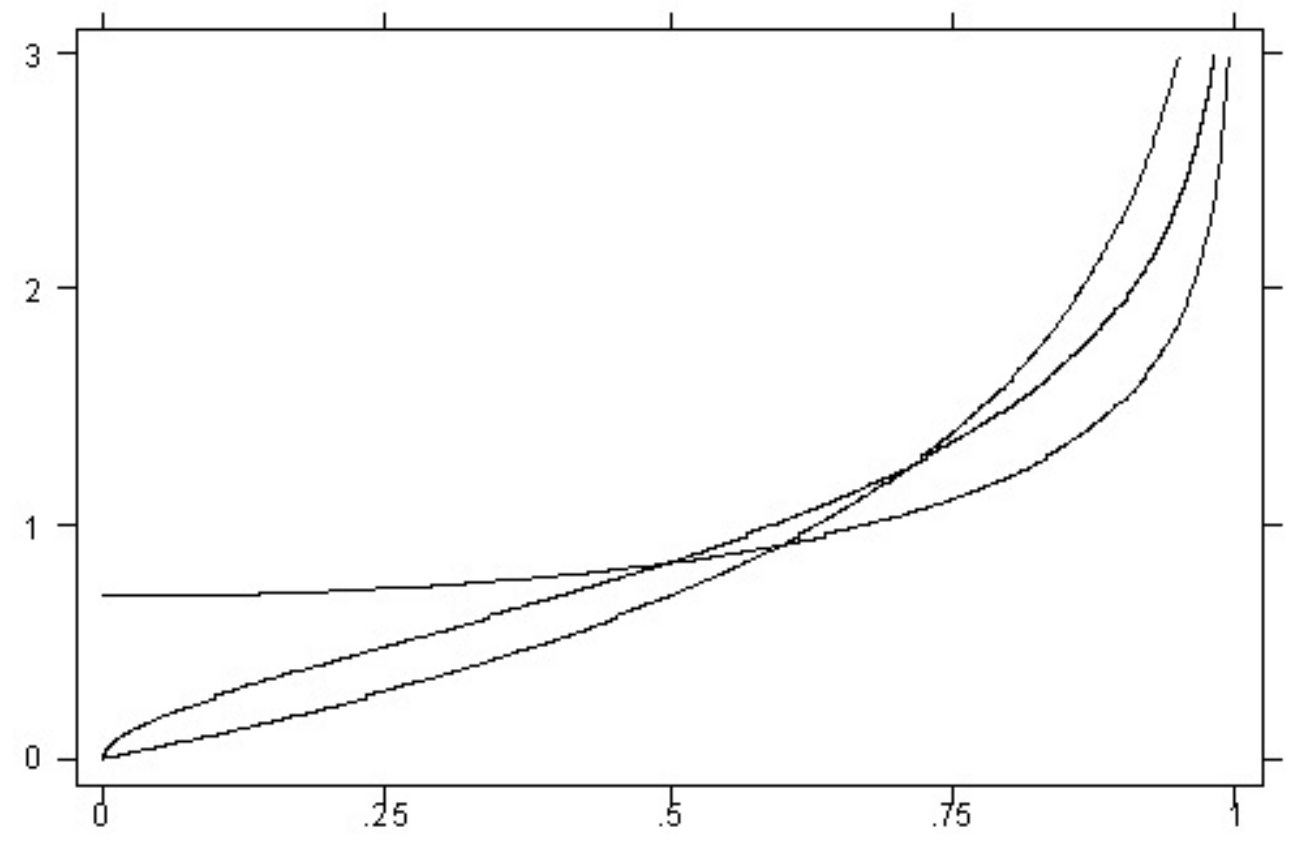

Figure 3. QF of the Standard Exponential, Erlang, and Ring(2)Exponential Variates (from bottom to top at $\alpha$ equals zero). 


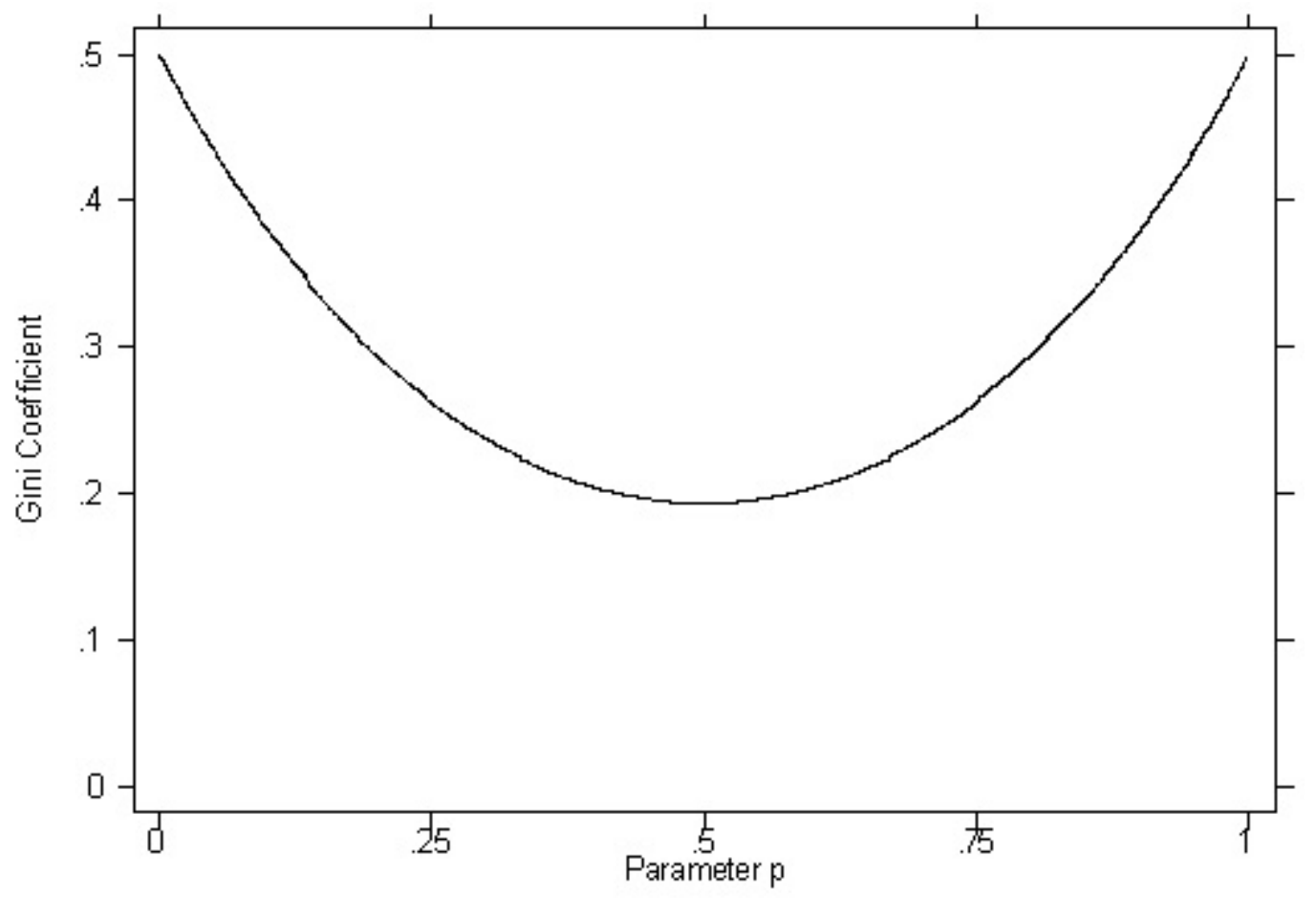

Figure 4. Gini Coefficient in the Mirror-Exponential 


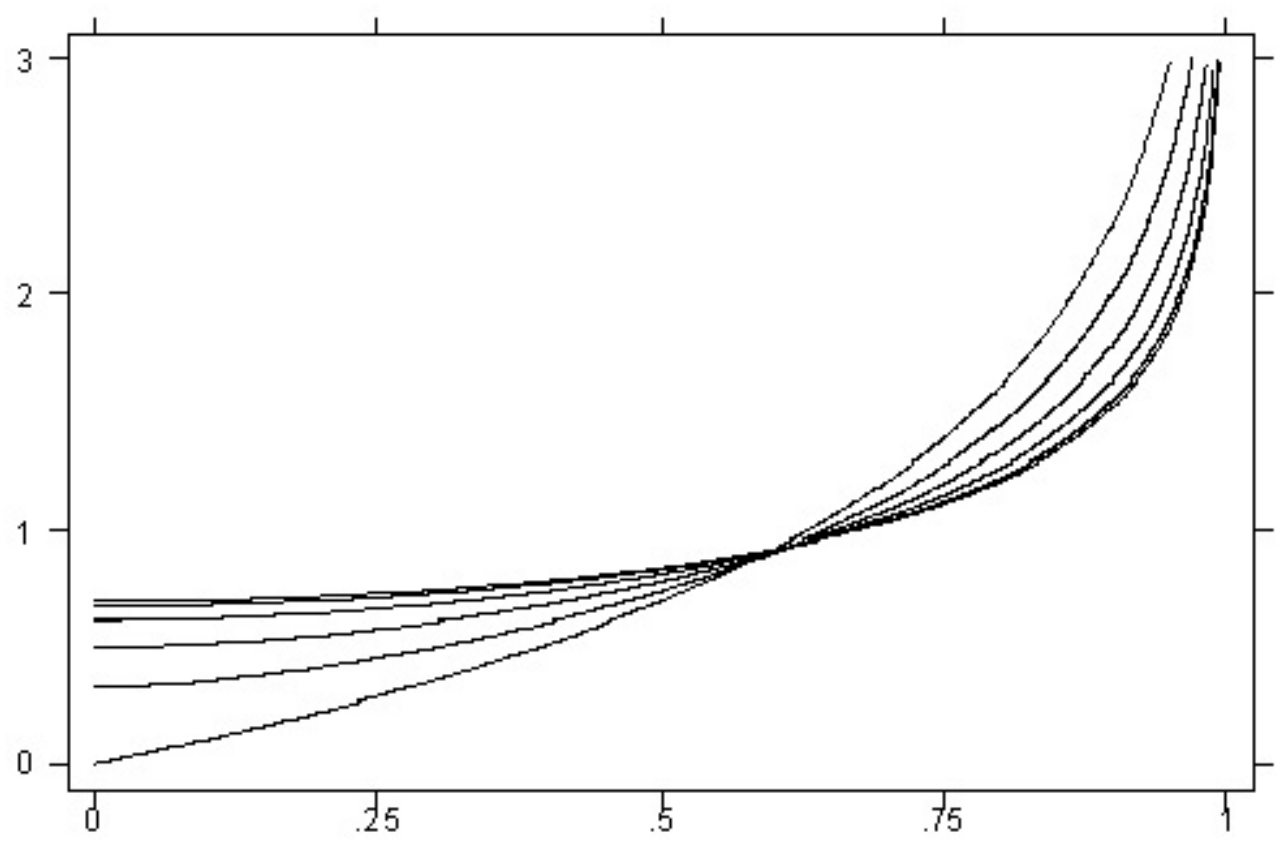

Figure 5. Quantile Function of Members of the Mirror-Exponential Family (with $p$ equal to $0, .1, .2, .3, .4$, and .5 , from bottom to top at $\alpha$ equals zero). The bottom and top members are, respectively, the standard exponential and the ring(2)-exponential. 


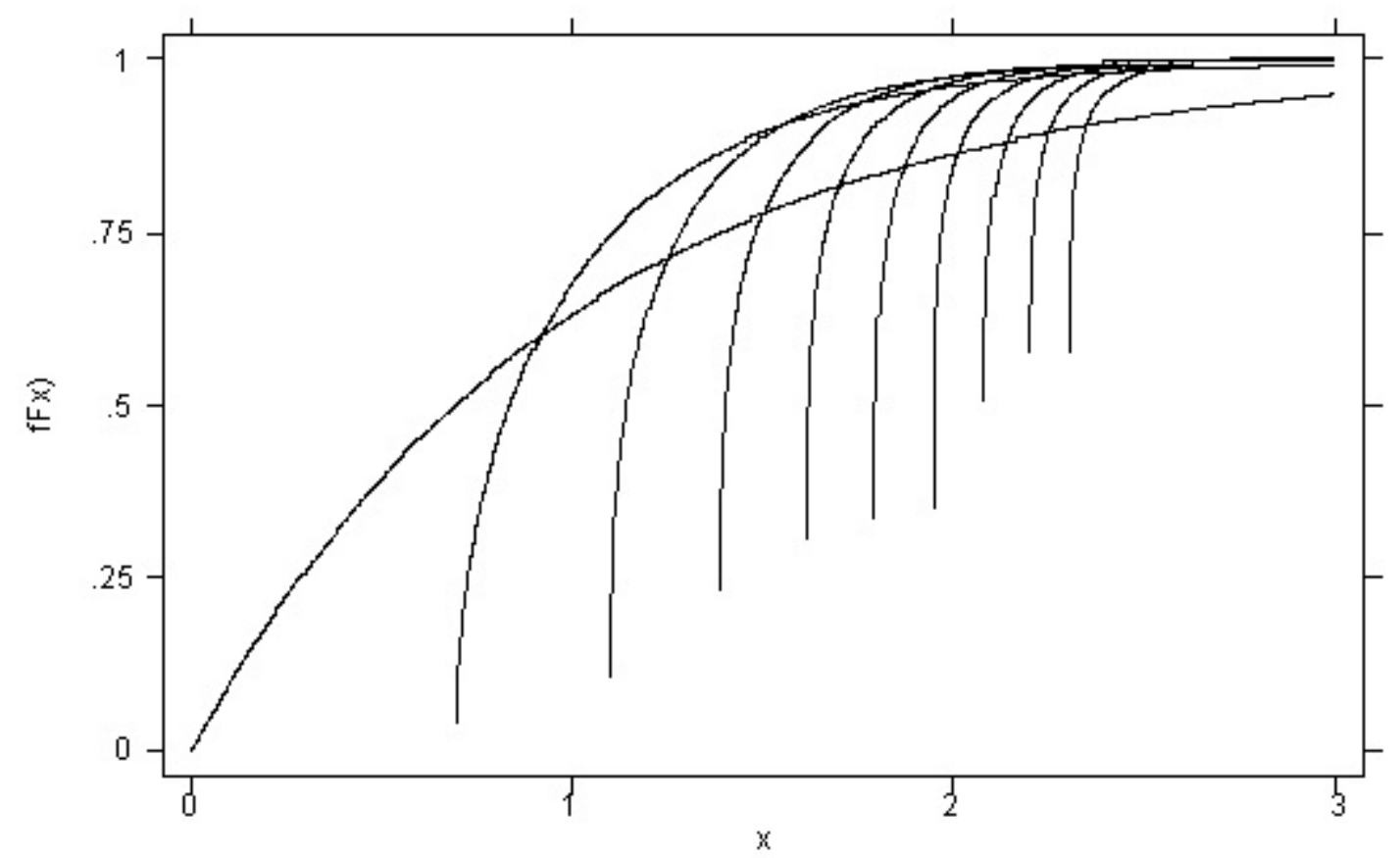

Figure 6. CDF of Members of the Ring-Exponential Family (with $k$ from 1 to 10, from left to right). The leftmost two members are the standard exponential and the ring(2)-exponential. 


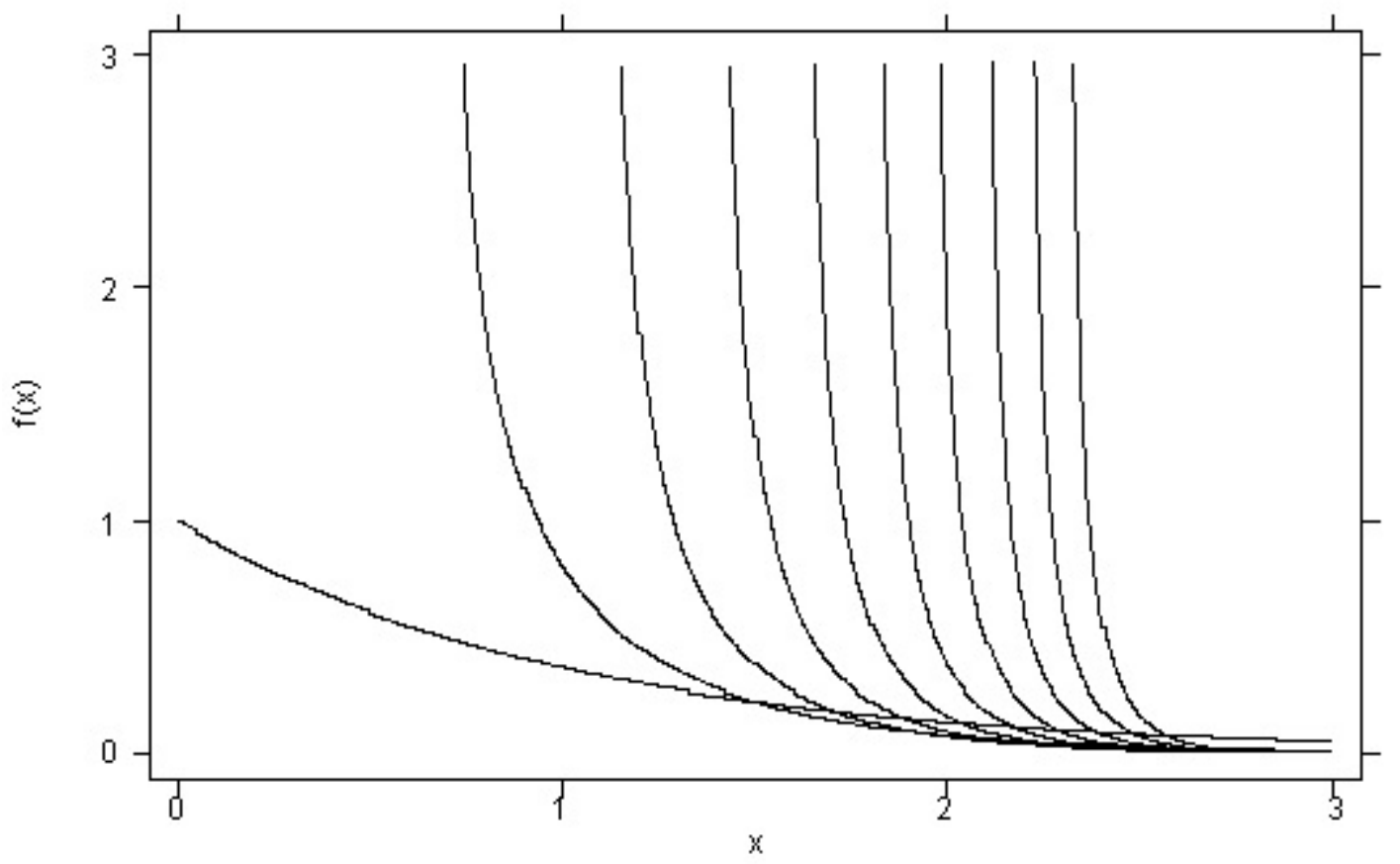

Figure 7. PDF of Members of the Ring-Exponential Family (with $k$ from 1 to 10, from left to right). The leftmost two members are the standard exponential and the ring(2)-exponential. 


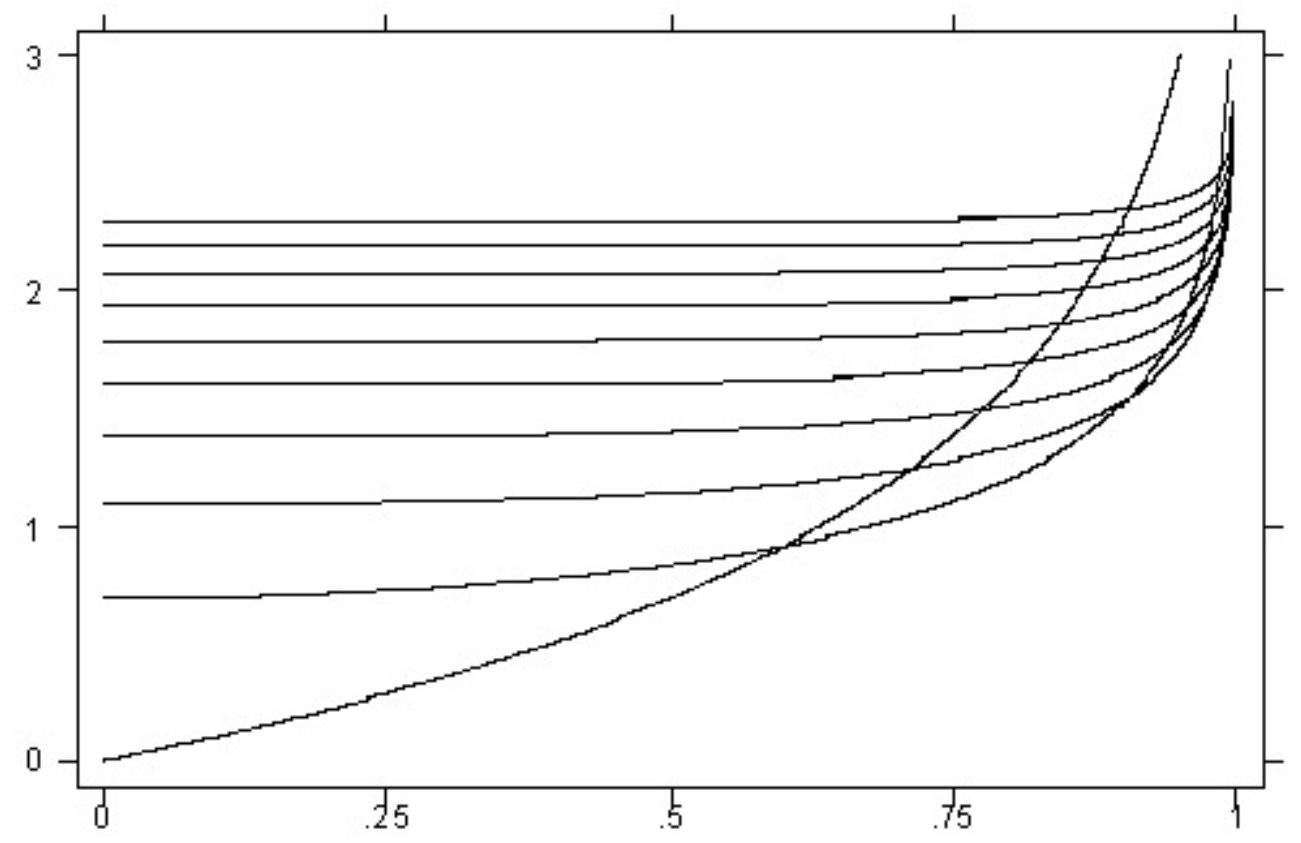

Figure 8. Quantile Function of Members of the Ring-Exponential Family (with $k$ from 1 to 10 , from bottom to top at $\alpha$ equals zero). The bottom two members are the standard exponential and the ring(2)-exponential. 


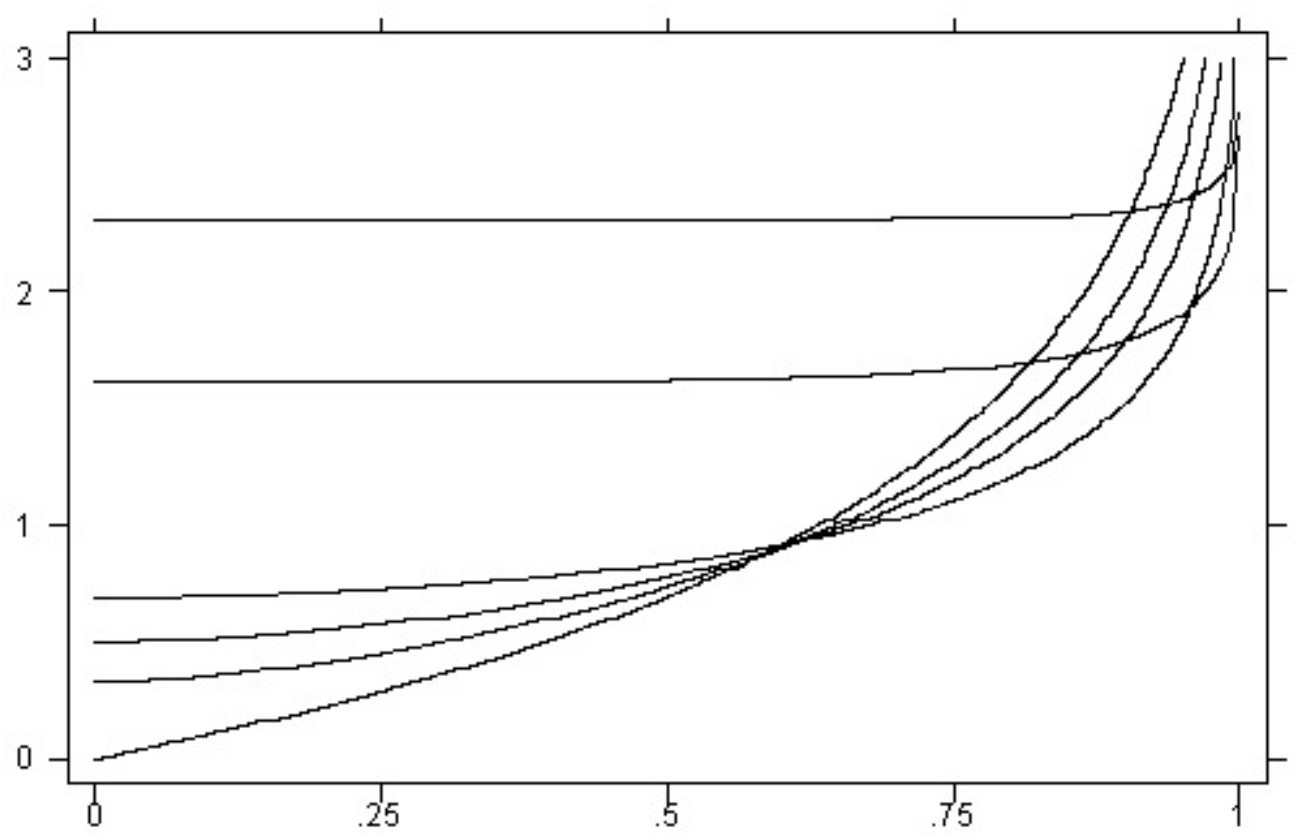

Figure 9. Quantile Function of Members of both the Mirror-Exponential and the Ring-Exponential Families. From bottom to top, the variates are the standard exponential (aka mirror-exponential with $p=0$ or 1 and ringexponential with $k=1$ ), two mirror-exponentials (with $p$ equal to .1 and .2 ), the ring(2)-exponential (aka mirror-exponential with $p$ equal to .5), and two ring-exponentials (with $k$ equal to 5 and 10 ). 